ARTICLE

\title{
The molecular basis of regulation of bacterial capsule assembly by Wzc
}

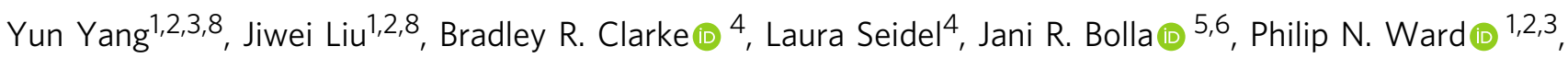
Peijun Zhang (i) ${ }^{2,7}$, Carol V. Robinson (1) ${ }^{5,6}$, Chris Whitfield (i) ${ }^{4 凶}$ \& James H. Naismith (1) ${ }^{1,2,3 凶}$

Bacterial extracellular polysaccharides (EPSs) play critical roles in virulence. Many bacteria assemble EPSs via a multi-protein "Wzx-Wzy" system, involving glycan polymerization at the outer face of the cytoplasmic/inner membrane. Gram-negative species couple polymerization with translocation across the periplasm and outer membrane and the master regulator of the system is the tyrosine autokinase, Wzc. This near atomic cryo-EM structure of dephosphorylated Wzc from E. coli shows an octameric assembly with a large central cavity formed by transmembrane helices. The tyrosine autokinase domain forms the cytoplasm region, while the periplasmic region contains small folded motifs and helical bundles. The helical bundles are essential for function, most likely through interaction with the outer membrane translocon, Wza. Autophosphorylation of the tyrosine-rich C-terminus of Wzc results in disassembly of the octamer into multiply phosphorylated monomers. We propose that the cycling between phosphorylated monomer and dephosphorylated octamer regulates glycan polymerization and translocation.

\footnotetext{
${ }^{1}$ Rosalind Franklin Institute, Harwell Campus, Harwell, UK. ${ }^{2}$ Division of Structural Biology, The University of Oxford, Oxford, UK. ${ }^{3}$ The Research Complex at Harwell, Harwell Campus, Harwell, UK. ${ }^{4}$ Department of Molecular and Cellular Biology, The University of Guelph, Guelph, ON, Canada. ${ }^{5}$ Physical and Theoretical Chemistry Laboratory, Department of Chemistry, South Parks Road, The University of Oxford, Oxford, UK. ${ }^{6}$ The Kavli Institute for Nanoscience Discovery, Oxford, UK. ${ }^{7}$ Electron Bio-Imaging Centre, Diamond Light Source, Harwell Science and Innovation Campus, Harwell, UK. ${ }^{8}$ These authors

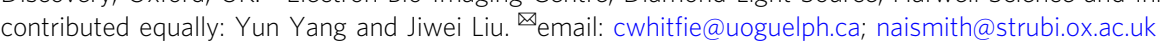


igh-molecular-weight extracellular polysaccharides (EPSs) play prominent roles in interactions between bacteria (pathogens, commensals, and symbionts) and their hosts (humans, livestock, and plants). Some EPSs are also important bioproducts in foods and other commercial applications. Although the structures of bacterial surface-associated and secreted polysaccharides are remarkably diverse, the majority are produced by a conserved and widely distributed assembly strategy (the Wzx-Wzy system) (reviewed in ref. ${ }^{1}$ ). The process begins with the synthesis of polyprenol diphosphate-linked oligosaccharide repeat units at the cytoplasmic face of the inner membrane (IM). The lipid-linked repeat units are then flipped across the IM by the Wzx flippase, where they act as substrates for the Wzy polymerase, an integral membrane protein with a catalytic site located at the external face of the IM. The subsequent assembly stages differ, depending on the bacterial species and the glycoconjugate, but all involve a polysaccharide co-polymerase (PCP) protein ${ }^{2}$ that is important for establishing the size distribution of the polymeric product. This is vital for the biophysical properties and biological functions of the polymers. In the production of capsular polysaccharides (CPS) or secreted EPSs in Gram-negative bacteria (Fig. 1a), Wzc (PCP-2a) proteins are thought to be the master regulator for both polymerization and translocation but the mechanism of regulation remains one of the most important questions in this field ${ }^{1}$. Wzc possesses a $\mathrm{C}$ terminal cytosolic protein tyrosine kinase (PTK) belonging to the BY-kinase family ${ }^{3}$, which contains Walker A and B motifs, as well as a tyrosine-rich tail presenting several residues for phosphorylation (reviewed in ref. ${ }^{2}$ ) (Fig. 1a). In contrast, the biosynthesis of lipopolysaccharide (LPS)-linked O-antigen polysaccharides by Wzx-Wzy pathways involves Wzz (PCP-1) homologs that lack a kinase domain and possess a shorter divergent periplasmic sequence compared to $\mathrm{Wzc}^{2,4}$. This reflects some functional differences; Wzz is important for regulating $\mathrm{O}$-antigen polymer chain length but plays no apparent role in the translocation of the final products ${ }^{4}$. Structural data are available for $\mathrm{Wzz}^{5-7}$ but not for full-length Wzc.

In Escherichia coli EPS assembly prototypes, the Wzc autokinase is paired with a cognate soluble protein tyrosine phosphatase (PTP) $)^{1,2}$. The structure of one PTP, Wzb, has been determined $^{8,9}$. The corresponding genes are found in the EPS biosynthesis genetic locus but many isolates also possess unlinked genes encoding an additional $\mathrm{Wzc}$ and $\mathrm{Wzb}$ homolog pair (designated Etk and Etp) ${ }^{10}$. Autophosphorylation of the Cterminal tyrosine-rich tail of $\mathrm{Wzc}$ is essential for polymerization of the EPS product ${ }^{11-13}$. Paradoxically, the removal of phosphates from Wzc by Wzb is also essential ${ }^{11-13}$. The requirement for both kinase and phosphatase activity has led to the hypothesis that the cycling between different Wzc phosphorylation states is critical for polymer production, rather than any one state ${ }^{13-15}$. This hypothesis holds in Gram-positive bacteria, which employ bipartite PCP-2b proteins resembling $\mathrm{Wzc}^{1}$. Crystal structures of isolated kinase domains from E. coli $\mathrm{Wzc}^{16}$ and S. aureus CapAB chimera $^{17}$ reveal octameric rings in which the nonphosphorylated C-terminal tail from one monomer is positioned in the active site of its neighbour. In contrast, Etk is not oligomeric in the presumed phosphorylated state ${ }^{18}$. The lack of a structure of the full-length protein where the kinase domains are in context is a major gap in functional understanding.

In Gram-negative bacteria, EPS must traverse the periplasm and outer membrane (OM) from the site of synthesis and this requires the octameric Wza translocon ${ }^{19,20}$ (Fig. 1a). Genetic data implicate specific Wza:Wzc interactions in EPS production and translocation $^{21}$ and oligomeric Wza:Wzc heterocomplexes were visualized in negatively-stained EM images ${ }^{22}$. The working hypothesis is that the periplasmic domain of Wzc engages with the periplasmic region of the Wza to form the structural machinery for translocation and EPS exits through the lumen of the translocon ${ }^{20}$. The absence of an OM in Gram-positive bacteria precludes this Wza interaction and CapA (for example) possesses a substantially reduced periplasmic domain.

Our understanding of the important physiological process of EPS biosynthesis and translocation is hindered by a lack of molecular insight into the master regulator, Wzc. Here we report the near-atomic structures of non-phosphorylated Wzc octamers from E. coli, in the apo- and ADP-bound states. We establish that the pattern of tyrosine phosphorylation controls the oligomerization state and demonstrate that periplasmic helical bundles are essential for function. We advance a mechanistic model for the EPS production system found across the bacterial kingdom, which is important for biotechnological applications and may offer options for targeted therapeutics.

\section{Results}

Non-phosphorylated full-length $\mathrm{K540M} \mathrm{Wzc}$ is an octameric protein. Native tyrosine-phosphorylated Wzc was purified but the protein eluted as a broad peak in gel filtration and negativelystained EM images showed heterogeneity in the samples (Supplementary Fig. 1a-c). Native protein mass spectrometry detected predominantly monomeric species and abundant phosphorylation adducts possessing 3, 4 or 5 phosphate groups (Fig. 1b). Further phosphoproteomic analysis suggested that the pentaphosphorylated species (5 P) was modified on tyrosines 708,713 , 715,717 and 718 . The strongest peak in tetra-phosphorylated (4 P) protein was modified on residues $708,713,715$ and 717 , but a species with phosphotyrosines at 708, 713,717 and 718 were also detected. The triple-phosphorylated (3 P) was more heterogeneous (Supplementary Fig. 2a, b).

To test the possibility that phosphorylation led to heterogeneity and aggregation of purified Wzc, a sample was dephosphorylated by treatment with phosphatase $\mathrm{Wzb}$ and then reapplied to the size exclusion column but it remained like the native protein (Supplementary Fig. 1d-f). Previous work showed that mutation of K540 in the Walker box abrogated Wzc phosphorylation ${ }^{13,17}$. In contrast to phosphorylated and enzymatically-dephosphorylated protein, purified non-phosphorylated $\mathrm{Wzc}^{\mathrm{K} 540 \mathrm{M}}$ was homogeneous, oligomeric (based on molecular mass in gel filtration) and yielded discrete octameric particles in negatively-stained EM images (Supplementary Fig. 1h-j). We re-examined the Wzb treated sample and collected 1787 cryo-EM images which were analysed by Topaz ${ }^{23}$ to identify 356,511 particles (Supplementary Fig. 1g). Through rounds of reference-free $2 \mathrm{D}$ classifications, 421 particles converged into clear views of an octameric Wzc (Supplementary Fig. 1g). In the raw micrographs, these particles (Supplementary Fig. 1g) resembled octamers of $\mathrm{Wzc}^{\mathrm{K} 540 \mathrm{M}}$. However, the small octameric population $(\sim 0.1 \%)$ in the $\mathrm{Wzb}$ treated sample made structure determination impractical, so we proceeded with $\mathrm{Wzc}^{\mathrm{K} 540 \mathrm{M}}$.

A cryo-EM structure of $\mathrm{Wzc}^{\mathrm{K} 540 \mathrm{M}}$ at $2.85 \AA$ resolution was determined with C1 symmetry (Supplementary Table 1, Supplementary Fig. 3a-e). Although the data were processed in $\mathrm{C}$, much of the structure displayed eightfold symmetry and the application of C8 symmetry improved the resolution to $2.30 \AA$ (Supplementary Fig. 3f, g). However, C8 symmetry breaks down for part of the periplasmic region, so local $\mathrm{Cl}$ refinement was used to complete the structure (Supplementary Fig. 3a, h, i). $\mathrm{Wzc}^{\mathrm{K} 540 \mathrm{M}}$ in complex with ADP was determined to $2.6 \AA$ with C8 symmetry (Supplementary Fig. $4 a-d$ ) and is essentially identical to the apo-structure, with the exception of additional density for ADP and $\mathrm{Mg}^{2+}$ (Supplementary Fig. 4e). Below, we focus on the higher resolution apo-structure. 
a

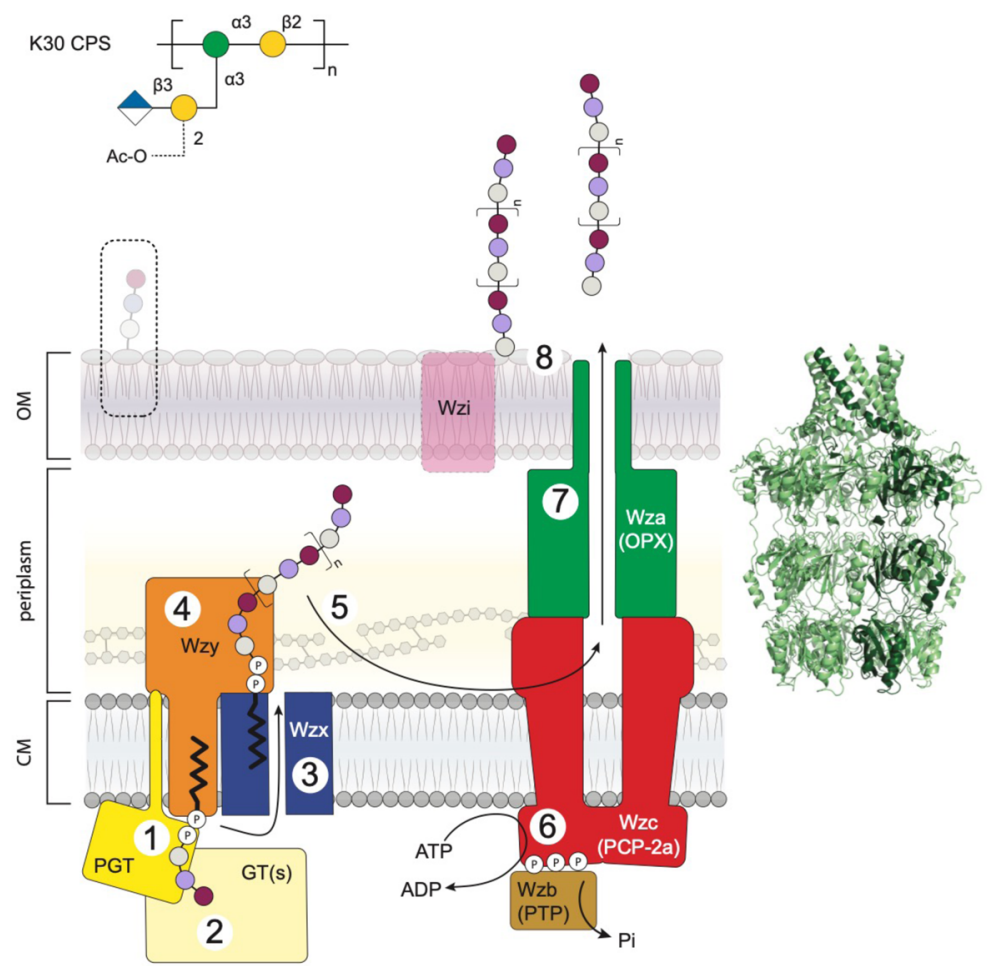

b

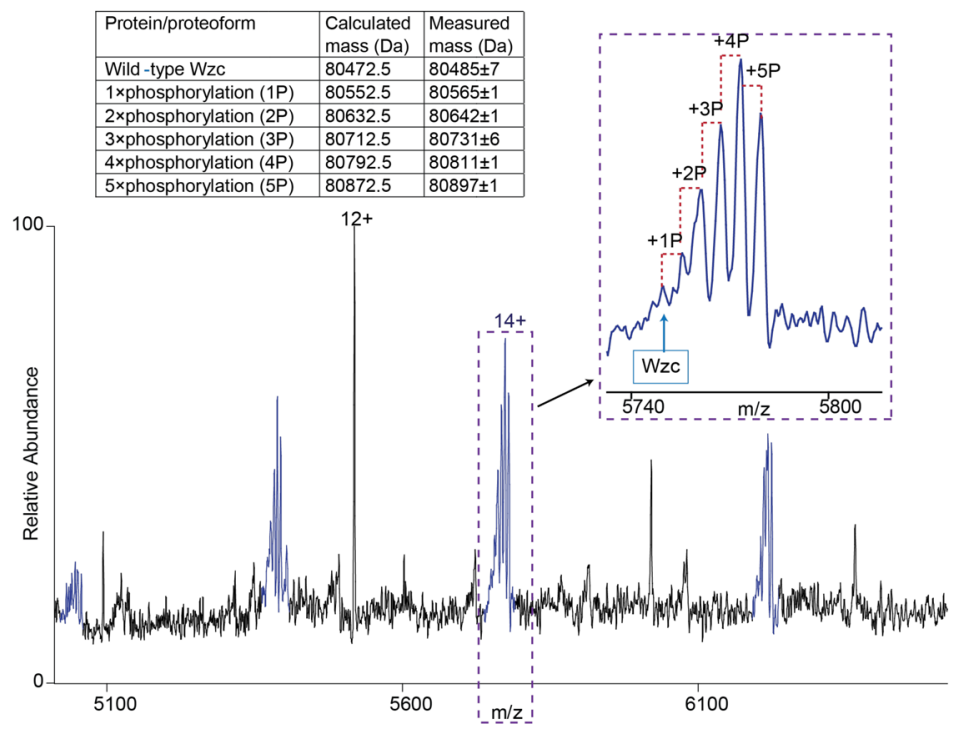

Fig. 1 Capsule assembly and Wzc. a Undecaprenyl diphosphate-linked oligosaccharide repeat units are synthesized by a phosphoglycosyltransferase (PGT; (1)) and serotype-specific glycosyltransferases (GT(s); (2)). These building blocks are flipped across the membrane by Wzx (3) and polymerized by Wzy (4) in a reaction regulated by the PCP-2a family autokinase, Wzc. Wzc cycles between phosphorylated and dephosphorylated (catalysed by Wzb) states for its function (6). The polymer is translocated across the outer membrane by Wza (7); the closed octameric structure of Wza' ${ }^{19}$ is shown on the right. Wza is also regulated by Wzc. In the prototype and some other species, Wzi supports the organization of translocated polymer into the surface-associated capsule structure (8) but this protein is absent from systems that produce secreted EPSs. In Gram-positive, bacteria step (7) is absent and nascent polymer is instead attached to peptidoglycan in the cell wall. In the absence of Wzc or Wza, short oligosaccharides representing one to a few repeat units are incorporated into LPS molecules in an off-pathway reaction (hatched box). The process has recently been reviewed ${ }^{1,4}$. The figure has been adapted from $^{1}$. b Purified, native phosphorylated Wzc appears only as a monomer (peak series highlighted in blue) in native mass spectrometry. The $14^{+}$charge state shows multiple phosphorylation (denoted P) states of Wzc with four sites being the most prevalent (inset). The theoretical and measured masses for all the observed species are shown in the table. Supplementary Fig. 2a identifies phosphopeptides. The peak series with a measured mass of $66,212 \pm 2 \mathrm{Da}$ was a contaminant protein. 

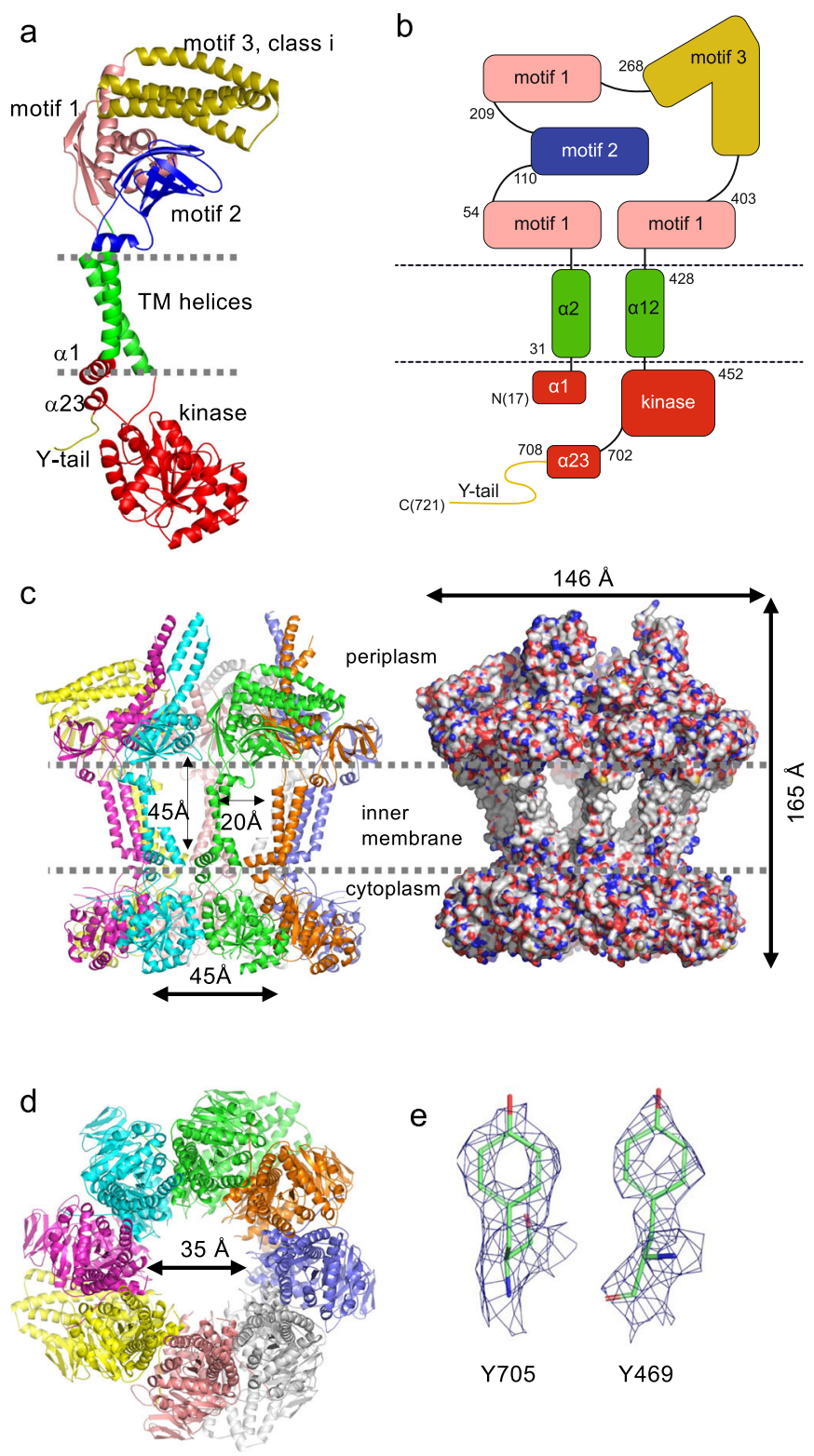

e
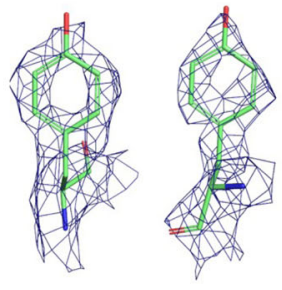

Y705

Y469

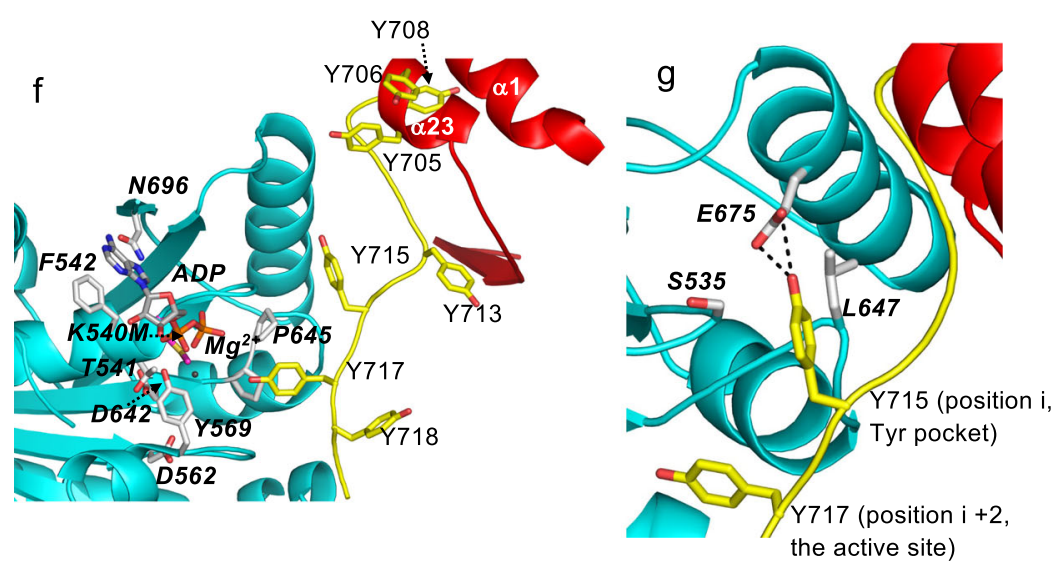

For ease of discussion, each protomer can be decomposed into three portions: the periplasmic region (residues 51-427), the transmembrane region (31-50, 428-447) and the cytoplasmic region (17-30, 448-721) (Fig. 2a, b). The Wzc ${ }^{\mathrm{K} 540 \mathrm{M}}$ octamer is $\sim 165 \AA$ high (cytosol to periplasm) and $146 \AA$ wide and possesses a large central cavity with side portals that open to the membrane bilayer (Fig. 2c, d). The high quality of the experimental map (Fig. 2e and Supplementary Fig. 3j-m) allows accurate assignment of the sequence to structural elements. The cytoplasmic region comprises a short N-terminal helix ( $\alpha 1$, Ile 17-Arg 30), the 
Fig. 2 The structure of $\mathbf{W z c}{ }^{\mathbf{K 5 4 0 M}}$ determined by single-particle cryo-EM. a The monomer of Wzc ${ }^{\mathrm{K} 540 \mathrm{M}}$ contains a cytoplasmic region (red), tyrosinerich tail (yellow), two transmembrane helices (green) and the periplasmic region (blue, salmon and ochre). b A schematic representation of the structure of Wzc K540M coloured as a The first residue of each structural block is shown. c Wzc K540M forms an octamer. The transmembrane helices are not closepacked and create portals to a large central cavity, clearly visible in the space fill representation. The structure has both a periplasmic and a cytoplasmic ring. $\mathbf{d}$ The $\mathrm{Wzc}{ }^{\mathrm{K} 540 \mathrm{M}}$ octamer viewed from the periplasm, reveals the periplasmic region also forms a ring-like arrangement. The central cavity is open to both the cytoplasm and lipid bilayer. Residues 65 to 84 could not be located experimentally and may occlude the entrance to the cavity from the periplasm. e The high quality of the EM map is illustrated by the holes for the aromatic residues. $\mathbf{f}$ The C-terminal tail from one monomer (coloured yellow and red) has residues labelled in normal text. Y717 is at the active site of the kinase domain from the other monomer (coloured cyan). Key kinase residues in the other monomer are labelled in bold italics. ADP and $\mathrm{Mg}^{2+}$ are shown and labelled. $\mathbf{g}$ With $\mathrm{Y} 717$ at the active site, Y715 sits in a pocket where it makes a hydrogen bond with $\mathrm{E} 675$ from the neighbouring subunit. A phosphorylated tyrosine at position $i$ would be disfavoured by size and charge. Disruption of binding at position $i$ could perturb binding at position $i+2$, the active site. Thus phosphorylation of Y715 seems most likely to follow (not precede) phosphorylation of Y717. Residues from the kinase domain are labelled in italics.

C-terminal kinase domain (Arg 452-Lys 701), and the tyrosine rich C-terminal tail (Ala 702-Lys 721) (Fig. 2b). The fold of the kinase domain was previously described in the crystal structure of the isolated cytoplasmic region from the closely related Wzc homolog from the colanic acid EPS biosynthesis system in E. coli (PDB 3LA6) ${ }^{16}$, the kinase domain from E. coli $\mathrm{Etk}^{18}$, and CapB from $S$. aureus ${ }^{17}$. Briefly, the kinase domain consists of central $\beta$-sheets sandwiched between two bundles of $\alpha$-helices. The C-terminal tyrosine-rich tail (residues 707-721) reaches across into the other neighbouring subunit, similar to the previous crystal structure of the isolated kinase domain ${ }^{16}$. The kinase active site has been described in detail for $\mathrm{Wzc}^{16}$, Etk ${ }^{18}$ and $\mathrm{CapB}^{17}$. The ADP complex confirms the active site location (Supplementary Fig. 4e) with key catalytic residues conserved (Supplementary Fig. 5a). Residues 418 to 474 of Wzc are not found in $S$. aureus CapB, but instead are part of CapA, the corresponding membrane-embedded partner needed to activate the kinase ${ }^{17}$. The C-terminal tail begins with $\alpha$-helix $\left(\alpha_{23}\right)$ (Ala 702 to Arg 707) which packs against the N-terminal $\alpha_{1}$ helix (Fig. 2a, b). The conserved tyrosine-rich region (Tyr 708, 713, 715 and 717) (Supplementary Fig. 5a) reaches into the active site of the neighbouring subunit with Y717 positioned at the active site (Fig. 2f). Y715 sits in a pocket, where it makes a hydrogen bond with E675 from the neighbouring subunit site (Fig. 2g). In contrast, the crystal structure of the isolated kinase domain ${ }^{16}$ has Y715 at the active site and Y713 is found in the pocket shown in Fig. 2g. In this crystal structure, the region corresponding to Ala 702-Arg 707 adopts a loop, rather than helix $\alpha_{23}$ seen in Wzc, possibly because the missing $\alpha_{1}$ helix is needed to stabilize $\alpha_{23}$. In CapB, the loop between $\beta_{20}$ of the kinase domain and the shorter tyrosine-rich tail adopts a very different arrangement ${ }^{17}$.

The transmembrane region of each Wzc monomer comprises two helices ( $\alpha_{2}$ K31 to L50 and $\alpha_{12}$ I428 to F447), which form an $\mathrm{X}$-like arrangement with multiple side-chain interactions between them (Fig. 2a). Together, the $\alpha_{12}$ helices create an inner ring in the octamer, while the eight $\alpha_{2}$ helices form an outer ring. The resulting arrangement of the eight transmembrane helical pairs is unusual; they are not closely packed, and this creates oval-shaped portals ( $20 \AA$ wide by $45 \AA$ high) located between the pairs of the helices (Fig. 2c).

The periplasmic region can be sub-divided into three distinct structural motifs (Fig. 2a, b). Motif 1 is formed by three noncontiguous stretches of the protein sequence (54-109, 209-267, 403-427) (Fig. 2b) and comprises four $\beta$ sheets and four $\alpha$ helices (Fig. 3a, Supplementary Fig. 6a). The structure suggests a periplasmic pore in Wzc but caution is required concerning this interpretation because it could conceivably be occluded by the conserved stretch of residues $65-84$, which are not located in the structure (Figs. 2d and $3 \mathrm{~b}$ ). The periplasmic ring principally creates contacts between motif 1 from each monomer (Fig. $3 \mathrm{~b}$ ). R410 of motif 1 is found at the interface, where it is within $4 \AA$ of the side chains of Q258 and R262 from the neighbouring monomer (Fig. 3c). R410 corresponds to R279 of Wzz (PDB $6 \mathrm{RBG})^{5}$ (Fig. 3d). Motif $2(110-206)$ is a jellyroll of 8 antiparallel strands with one a helix (Fig. 3a, Supplementary Fig. 6a). In the octamer, motif 2 is positioned radially and makes no contact with the neighbouring monomers (Fig. 3b). Within each monomer, $\alpha_{5}$ packs against the transmembrane helix $\alpha_{2}$ (Fig. 3a).

In contrast to motifs 1 and 2, motif 3 (268-402) is not well ordered in the C8-averaged map (Supplementary Fig. 3f, g) and working in $\mathrm{C} 1$ identified three discrete structural arrangements (Fig. 3b, Supplementary Fig. 7a-e). This is an unusual break in symmetry. We designate the three possible arrangements as class $\mathrm{i}$, ii and iii. In the most common particle, class $\mathrm{i}$ is found in three monomers (chains $\mathrm{A}, \mathrm{D}$ and $\mathrm{F}$ ) with all motif 3 residues visualised; this permits an almost complete model of the Wzc monomer (Supplementary Table 1, Fig. 2a, Supplementary Fig. $7 a-c)$. Class $i$ is a four coiled-coil helical bundle $\left(\alpha_{8}-\alpha_{11}\right)$, composed of two helical pairs $\left(\alpha_{8}-\alpha_{11}\right.$ and $\left.\alpha_{9}-\alpha_{10}\right)$. The helical axes are arranged perpendicular to the central eightfold axis of Wzc and are folded down onto the surface of motif 1 (Figs. $2 \mathrm{a}$ and $3 b)$. Only a portion of $\alpha_{8}$ and $\alpha_{11}$ could be traced in class ii (chains B, C, G and $\mathrm{H}$ ), where the helical axes are arranged approximately parallel to the eightfold axis (Fig. 3a, b and Supplementary Fig. 7a, b, d-f). This arrangement is found as a pair in neighbouring subunits, with the helices from one subunit stacking against the other (Fig. 3b). We were unable to identify any density for motif 3 in chain $E$ (class iii) (Fig. 3b, Supplementary Fig. 7b). The striking differences in motif 3 point to conformational flexibility.

Approximately two-thirds of the surface area buried by the formation of octamer occurs in the cytosolic region (Fig. 3e), with the remainder arising from contacts between motif 1 of the periplasmic domain (Fig. 3b). The interaction between the Cterminal tyrosine-rich tail and the kinase active site of each neighbouring subunit (Fig. 3e) buries around $440 \AA^{2}$ surface area (of total $1200 \AA^{2}$ buried).

The transmembrane helices and most of motif 1 in Wzc can be superimposed with $\mathrm{Wzz}(\mathrm{PDB} 6 \mathrm{RBG})^{5}$ with a root mean square deviation (rmsd) $2.1 \AA$ over $130 \mathrm{Ca}$ atoms (Fig. $3 \mathrm{f}$ ). Wzz lacks the kinase domain and periplasmic motif 2. In Wzz, motif 3 is replaced by an extended helical bundle with a very different arrangement (Fig. 3f). The helical barrel evident in the Wzz octamer $^{5-7}$ is not found in Wzc (Supplementary Fig. 6b). Wzz and Wzc share the open arrangement of the transmembrane helices and the periplasmic ring formed by motif 1 (Supplementary Fig. 6b, c). However, the interfaces between motif 1 in Wzz bury approximately twice as much surface area as the corresponding motif in Wzc. In Wzc, the presence of motif 2 changes the local structure of motif 1 at the motif 1 contact interface; in Wzz this region makes extensive intersubunit contacts $^{5-7}$. The unfavourable R410-R262 interaction of Wzc is 
a
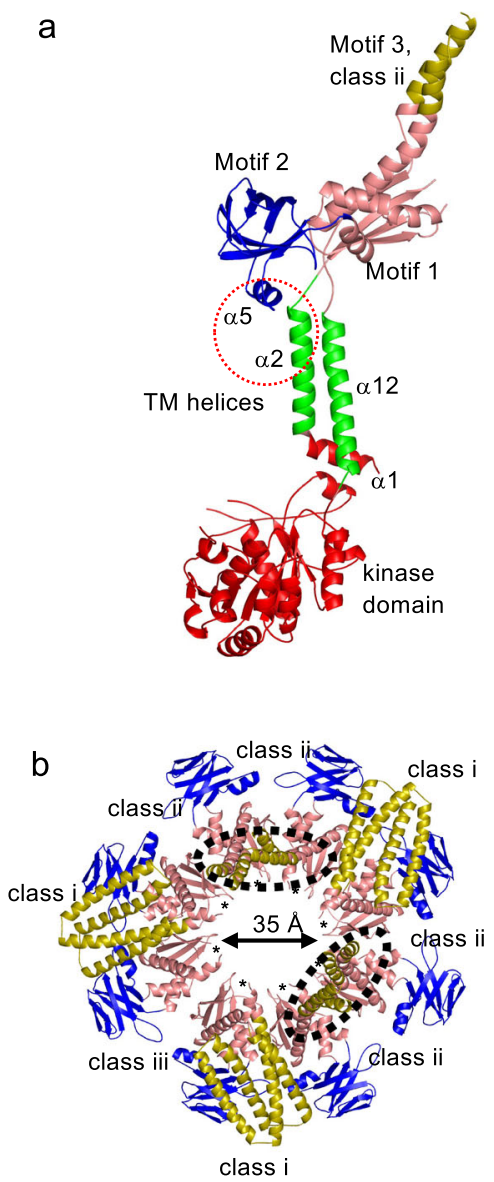

C

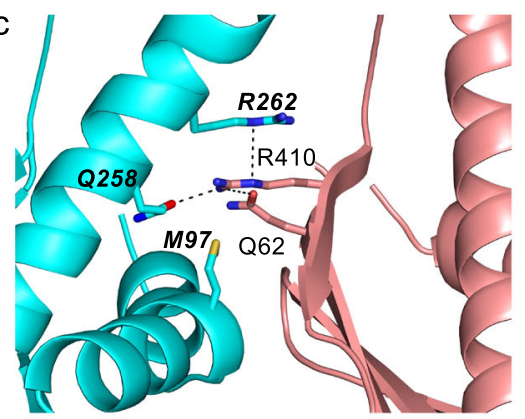

e

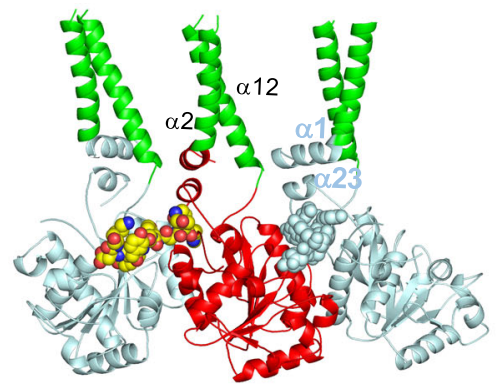

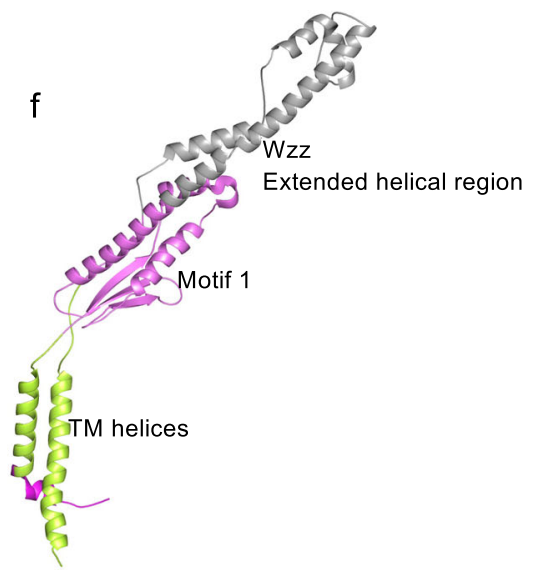

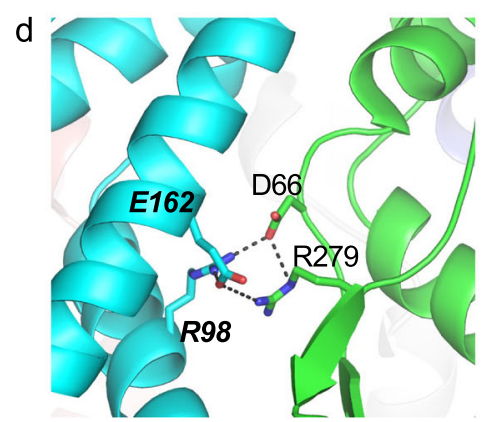

also present in Wzz (R279- R98) but there are compensating salt bridges with D66 and E162 in Wzz (Fig. 3d).

The role of the tyrosine-rich tail. In mass spectrometry, the native protein was shown to be phosphorylated and predominantly monomeric, echoing reports of the behaviour of isolated kinase domains ${ }^{18}$ (Fig. 1b). Systematic Tyr to Phe mutations established that the C-terminal tyrosine residues were essential for capsule production, but no single tyrosine was essential, nor was any single tyrosine sufficient ${ }^{14,24}$. Reasoning the negatively charged Glu would mimic negatively charged pTyr (phosphotyrosine) and create a protein resembling a locked phosphorylated state, several Tyr-to-Glu mutants were constructed in $\mathrm{Wzc}^{\mathrm{K} 540 \mathrm{M}}$. With the exception of $\mathrm{Y} 706 \mathrm{E}$, each single Tyr to Glu replacement, as well as double (717 and 718) and triple 
Fig. 3 The periplasmic and cytoplasmic rings of Wzc. a The class ii arrangement of motif 3 is shown. This is very different to the class $i$ arrangement shown in Fig. 2a, suggesting motif 3 is dynamic. Motif 2 makes contact with $\alpha_{2}$ thereby forging a structural connection between the periplasm and cytoplasm. The colour scheme is as Fig. 2a. b The periplasmic ring of Wzc, using the colour scheme in Fig. 2a. The ring is predominantly held together by contacts between motif 1 of neighbouring monomers. The class ii arrangement is found as a pair where the helices from one monomer stack with the other monomer (circled). In the class iii arrangement, the helices are almost entirely disordered. c R410 is at the interface between the periplasmic domains of Wzc, where it makes interactions with Q62 and from the neighbouring subunit R262 and Q258 (bold italics). Interactions closer than $4 \AA$ are shown as dashed lines. The interface in Wzz is shown in (d). d The interface in Wzz (PDB 6RBG) ${ }^{5}$, corresponding to that shown for Wzc in (c). In Wzz, R279 (equivalent to R410 in Wzc) is at the interface between the periplasmic domains, where it makes interactions with D66 and from the neighbouring subunit R98 and E162 (denoted bold italics). Interactions close to $4 \AA$ are shown as dashed lines. e The cytoplasmic ring of Wzc K540M is held together by interactions between the neighbouring kinase domain and the tyrosine-rich C-terminus which is a target for phosphorylation (shown as a space fill sphere) that reach the active site of the neighbouring monomer. f Full-length Wzz (PDB 6RBG) ${ }^{5}$, shares the same arrangement of TM helices (pale green) and part of motif 1 (pale pink). The extended helical region (grey) in Wzz does not resemble any class of motif 3 in Wzc. Thus, the kinase domain, motif 2 and motif 3 of Wzc are all unique features. The octameric assembly of Wzz is shown in Supplementary Fig. 6b, c.

(715, 717 and 718) mutants formed octamers, as judged by EM (Supplementary Fig. 8a). We term this set of Y to E mutants "octamer capable". A crude analysis of the percentage of octamers on the EM grid shows that as the number of $\mathrm{Y}$ to $\mathrm{E}$ substitutions increased, the portion of particles that were octameric reduced (Supplementary Fig. 8a). The capacity of these mutants to sustain autophosphorylation and polysaccharide production was assessed by constructing the same mutations in native (phosphorylationproficient) Wzc protein. As expected, western blotting and mass spectrometry showed the amount of phosphorylation decreased with increasing numbers of replaced tyrosine residues (Fig. $4 \mathrm{~b}$, Supplementary Fig. 2c). Mass spectrometry showed these mutants are found as (with decreasing extent) phosphorylated monomers (Supplementary Fig. 2c), similar to the native protein. The "octamer capable" Y to E mutants (Supplementary Fig. 8a) all supported capsule production in vivo when introduced into the native $w z c$ background (Supplementary Fig. 8b, c). Structural analysis suggests the Y706E mutant would perturb the interaction of $\alpha_{1}$ and $\alpha_{23}$, consistent with the observed functional impairment in single and double mutants involving Y706.

The introduction of four or more Glu residues resulted in severe defects in capsule production (Fig. 4a, Supplementary Fig. 8f). Phosphorylation of these proteins was not detected in whole-cell lysates (Fig. 4a) but this was attributed to reduced levels and limits of detection in lysates; phosphorylation was confirmed with the purified proteins (Fig. 4b, Supplementary Fig. 2c). A mutant with seven Glu residues did not support capsule production or autophosphorylation, as predicted by previous work ${ }^{24}$ (Supplementary Fig. 8f).

Analysis of cryo-EM images of $\mathrm{Wzc}^{\mathrm{K} 540 \mathrm{M}}$ with four Glu

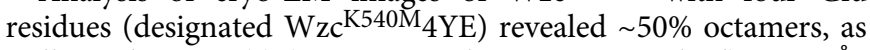
well as other state(s) (Fig. 4c, Supplementary Fig. 9b-d). A $2.8 \AA$ -

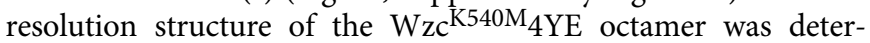
mined using the same approach as $\mathrm{Wzc}^{\mathrm{K} 540 \mathrm{M}}$ (Supplementary Fig. 9a-j). Overall, this structure has similar octameric (rmsd 3.1 $\AA$ over $4520 \mathrm{Ca}$ atoms) and monomeric (rmsd of $2.5 \AA$ over 651 $\mathrm{Ca}$ atoms) arrangements seen in $\mathrm{Wzc}^{\mathrm{K} 540 \mathrm{M}}$ (Supplementary Fig. 9e). The core kinase domain is unchanged between the structures (rmsd $0.4 \AA$ for Ca atoms 452-697) but the tyrosinerich tail has undergone a large change that now places $\mathrm{Y} 708$ at the active site (compared Y717 in Wzc ${ }^{\mathrm{K} 540 \mathrm{M}}$ ) (Fig. 4d). Excluding the C-terminal tail and re-calculating the superpositions for monomer and octamer yields rmsd values of $1.8 \AA$ and $2.3 \AA$ respectively. The change in the tail results in a reduction of the buried surface area that holds the octamer together. In

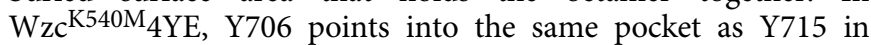
$\mathrm{Wzc}{ }^{\mathrm{K} 540 \mathrm{M}}$ (Fig. 2g) but this residue no longer makes the hydrogen bond with E675. In $\mathrm{Wzc}^{\mathrm{K} 540 \mathrm{M}} 4 \mathrm{YE}$, helix $\alpha_{23}$ is unwound, and the resulting loop no longer makes any contacts with helix $\alpha_{1}$, whose position is altered as a result (Fig. $4 \mathrm{~d}$ ). The structure supports a model where the Tyr residues located Cterminal to Y708 have been phosphorylated, consistent with the observed multiple phosphorylation of the C-terminal peptide $^{13-15}$.

The secondary structures of motif 1 and motif 2 in the

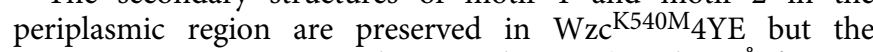
structure comparison reveals some changes ( $r m s d 0.8 \AA$ for 207 $\mathrm{Ca}$ atoms). Motif 3 still possesses the same three structural arrangements seen in $\mathrm{Wzc}^{\mathrm{K} 540 \mathrm{M}}$ but only two (not three) monomers have the fully ordered class i arrangement (rmsd of $0.4 \AA$ for $133 \mathrm{Ca}$ atoms). The larger rmsd value for the monomer and octamer, compare to the individual regions, reflects shifts in the relative positions of the regions. This can be visualised by superimposing the core kinase domains of monomer for $\mathrm{Wzc}^{\mathrm{K} 540 \mathrm{M}}$ and $\mathrm{Wzc}^{\mathrm{K} 540 \mathrm{M}} 4 \mathrm{YE}$, which shows a rigid body rotation of $11^{\circ}$ at the other end of the molecule (Fig. 4e). Superposition using all the $\mathrm{Ca}$ atoms in octamer reveals shifts in the periplasmic helices, particularly those in class ii (Fig. 4f).

pY718 was observed in some peptides of the native protein (Supplementary Fig. 2a) but it is not a conserved residue (Supplementary Fig. 5a). An additional set of multiple Tyr to Glu mutants was constructed in order to test whether Y718 itself played a role and revealed that Y718 has no functional significance (Supplementary Fig. 8a, d, e).

The role of motif 3 . The structures of the helical bundles appear to be dynamic within the octamer (resulting in three possible arrangements). Comparing $\mathrm{Wzc}^{\mathrm{K} 540 \mathrm{M}}$ vs $\mathrm{Wzc}^{\mathrm{K} 540 \mathrm{M}_{4} \mathrm{YE} \text { revealed }}$ the distribution of arrangements changed, suggesting the helical bundles are particularly sensitive to changes elsewhere in the structure. However, we are unable to exclude the possibility that this difference is due to a relatively smaller dataset of $\mathrm{Wzc}^{\mathrm{K} 540 \mathrm{M}} 4 \mathrm{YE}$ compared with $\mathrm{Wzc} \mathrm{K}^{540 \mathrm{M}}$. To further probe the functional significance of the helical bundles, a construct lacking motif 3 ( $\Delta$ Motif3) was engineered in either $\mathrm{Wzc}$ or $\mathrm{Wzc}^{\mathrm{K} 540 \mathrm{M}}$ backgrounds. As expected, purified $\mathrm{Wzc}^{\mathrm{K} 540 \mathrm{M}} \Delta \mathrm{Motif} 3$ formed octamers, same as Wzc ${ }^{\mathrm{K} 540 \mathrm{M}}$ (Fig. 5a). However, the Wzc $\Delta \mathrm{Mo}$ tif3 protein was unable to support capsule production establishing these helices are key to function, despite the preservation of its autokinase activity (Fig. 5b).

\section{Discussion}

Wzc participates in the production of critical CPS virulence factors in prominent multidrug-resistant ESKAPE pathogens Klebsiella pneumoniae ${ }^{25}$ and Acinetobacter baumannii ${ }^{26}$. This makes Wzc a candidate for antibacterial development to counter these and other pathogens. In the $E$. coli K30 prototype, Wzc (PCP-2a) proteins regulate polymerization, as deletion of $w z c$ results in no accumulation or synthesis of any high-molecular- 


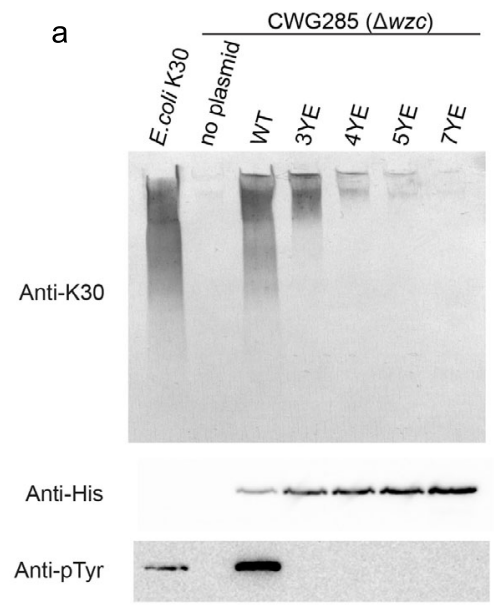

b

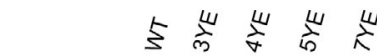

Anti-pTyr

Anti-His

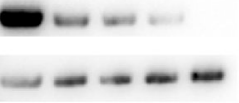

C
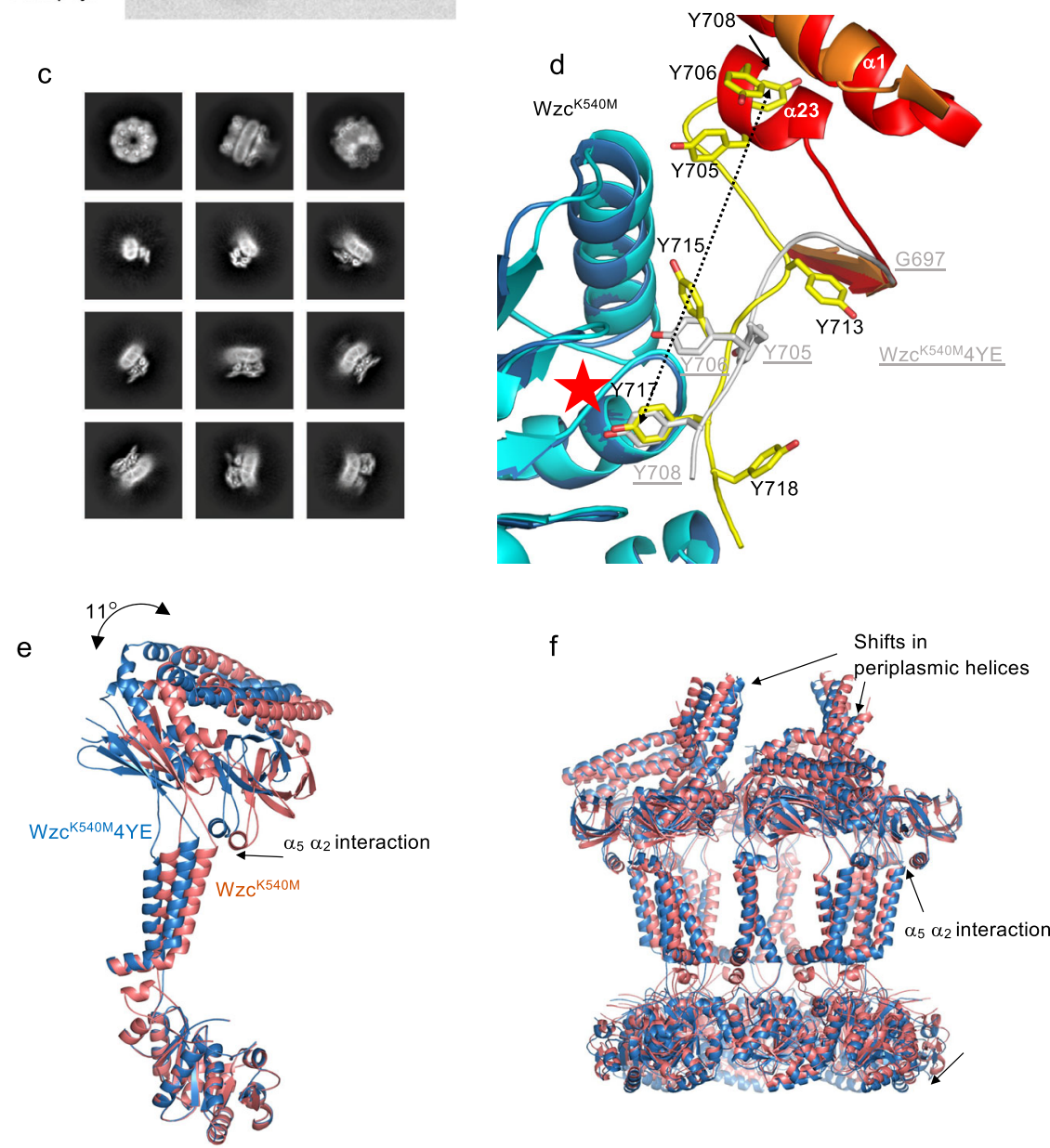

weight CPS $^{1,13}$. An explicit link between Wzc and polymer chain length was also reported for $E$. coli colanic acid ${ }^{14}$. A gain-offunction mutation in $A$. venetianus RAG1 wzc, (equivalent to $\mathrm{R} 410 \mathrm{~L}$ in $\mathrm{Wzc}$ ) leads to a hyper mucoid phenotype and increased apparent molecular weight (and potential value) of the commercial biopolymer known as emulsan ${ }^{27}$.

Since Wzc and its O-antigen biosynthesis counterpart, $\mathrm{Wzz}^{5}$, both regulate the polymerase Wzy, this aspect of their function(s) would be expected to reflect some structural conservation. Both proteins share most of motif 1 which forms an octameric ring and both have a large chamber in the membrane formed by the transmembrane helices (Supplementary Fig. 6b, c). The chamber offers a plausible location for Wzy and the portals would allow

free exchange of lipid-linked oligosaccharides substrates. This location would imply contact between Wzy and $\alpha_{12}$ of Wzc and some experimental support for this proposal comes from mutants of the equivalent helix of Wzz that are known to alter Wzy behaviour $^{28}$.

Wzc possesses a cytoplasmic autokinase domain and two periplasmic sub-domains (motifs 2 and 3) that are absent in Wzz. Non-phosphorylated Wzc is an octamer held together by the interaction between tyrosine-rich C-terminal peptide of one monomer and the active site of the kinase domain of neigh-

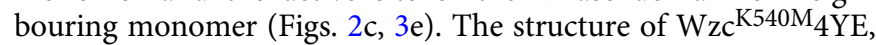
chosen to mimic a phosphorylated state, showed a significant conformational change in the tyrosine tail, which would be 
Fig. 4 The effect of phosphorylation. a Western immunoblot of whole cell lysates probed with anti-K30 antiserum to detect cell surface polysaccharides (top panel), the anti-His antibody for expression (middle panel) and anti-pTyr antibody for pTyr (bottom panel). E. coli K30 (strain E69) is used as a positive control. E. coli CWG285 (wzC mutant) is used as a negative control. WT, 3YE, 4YE, 5YE and 7YE represent plasmids encoding wild-type WzC, Y715E/Y717E/Y718E mutant, Y713E/Y715E/Y717E/Y718E mutant, Y708E/Y713E/ Y715E/Y717E/Y718E mutant and Y705E/Y706E/Y708E/Y713E/ $\mathrm{Y} 715 \mathrm{E} / \mathrm{Y} 717 \mathrm{E} / \mathrm{Y} 718 \mathrm{E}$ mutant respectively. Bacteria were grown for $16 \mathrm{~h}$ without L-arabinose induction to obtain Wzc expression levels similar to chromosomal copy. Uncropped gels with molecular weight markers are shown in Supplementary Fig. 8f. Experiments were performed in biological triplicate and were consistent, a representative experiment is shown. $\mathbf{b}$ Western immunoblot of purified Wzc proteins probed with anti-pTyr antibody and anti-His antibody is labelled as above. The phosphorylation of 3YE,4YE,5YE not visible in (a) is attributed to sensitivity. Uncropped gels with molecular weight markers in shown in Supplementary Fig. 10a. Experiments were performed in technical triplicate. c Representative 2D class averages of Wzc ${ }^{\mathrm{K} 540 \mathrm{M}} 4 \mathrm{YE}$ (mimicking phosphorylation of tyrosines) showing octamer and non-octameric particles (box size $312 \AA$ ). $\mathbf{d}$ A superposition of the kinase active site of

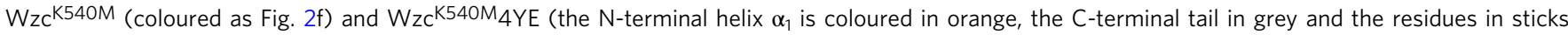

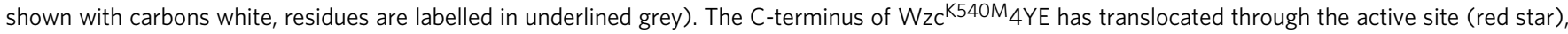
the shift in $Y 708$ is shown as an arrow. As a consequence of the movement, $\alpha_{23}$ has been entirely unwound which in turn perturbs $\alpha_{1}$. e Superposition (using the kinase domain) of the monomers of Wzc ${ }^{\mathrm{K} 540 \mathrm{M}}$ (coloured salmon) and $\mathrm{Wzc}^{\mathrm{K} 540 \mathrm{M}} 4 \mathrm{YE}$ (dark blue) shows a structural shift in the protein with a relative rotation in the periplasmic domains, these changes are transmitted through the transmembrane helices. $\mathbf{f}$ Superposition of the octamer of $\mathrm{Wzc}^{\mathrm{K} 540 \mathrm{M}}$ (coloured salmon) and $\mathrm{Wzc}^{\mathrm{K} 540 \mathrm{M}} 4 \mathrm{YE}$ (dark blue) reveals shifts in the periplasmic helices.

a

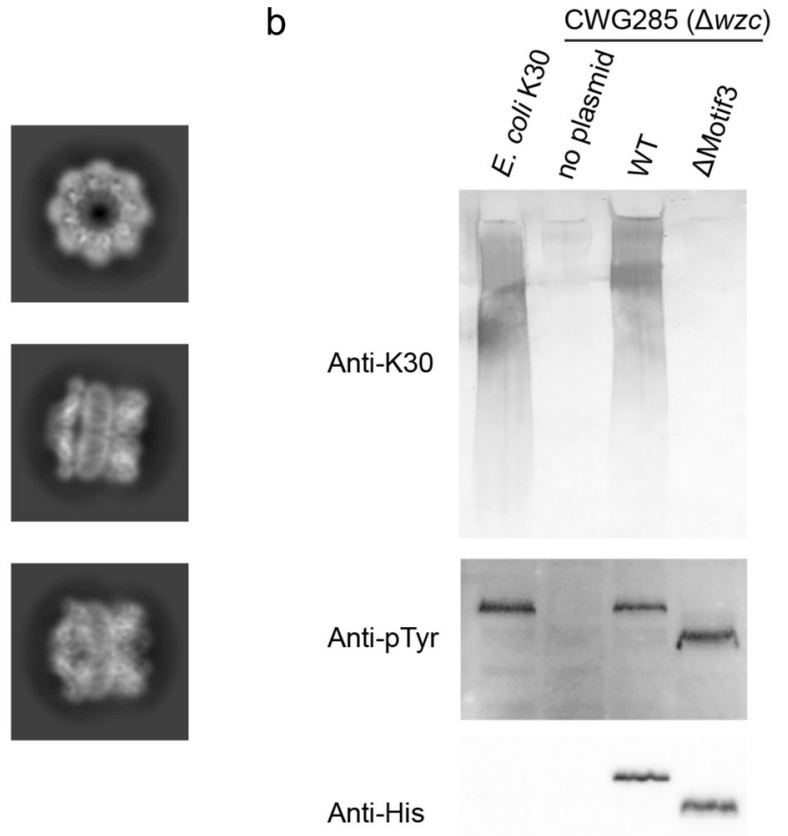

Fig. 5 Function of $\mathbf{W z c}$ periplasm motif 3. a Representative $2 D$ class averages of Wzc K540M $\Delta$ Motif3 show the protein forms an octamer. CryoEM data were collected on Glacios (Thermo Fisher). Box size, $248 \AA$. b Western immunoblot of whole cell lysates probed with anti-K30 antiserum to detect cell surface polysaccharides (top panel), the anti-pTyr antibody for pTyr (middle panel) and anti-His 5 antibody for expression (bottom panel). Bacteria were grown for $16 \mathrm{~h}$ without $\mathrm{L}$-arabinose induction to achieve amounts of Wzc similar to those from chromosomal copies. Uncropped gels with molecular weight markers are shown in Supplementary Fig. 10b. Experiments were performed in biological triplicate and were consistent, a representative experiment is shown.

predicted to weaken the octamer (Fig. 4c, d, Supplementary Fig. 9b-d). There is some evidence for different oligomeric states (other than octamer) in Wzc ${ }^{\mathrm{K} 540 \mathrm{M}} 4 \mathrm{YE}$ (Supplementary Fig. 9d) but none were amenable to structural determination. The introduction of one further replacement (5YE) resulted in the complete loss of the octamer form (Supplementary Fig. 8a).

Systematic mutagenesis indicated that progressive phosphorylation destabilises the octamer (Supplementary Fig. 8a) until the point where four conserved tyrosines (Y708, Y713, Y715, Y717) are phosphorylated. This species represents the tipping point where the stable octamer is unable to form and the protein found as monomers. Structural data would suggest phosphorylation typically starts at Y717 (Fig. 2f). In Wzc $\mathrm{K}^{\mathrm{K} 40 \mathrm{M}}$, Y715 sits in a pocket (Fig. $2 \mathrm{~g}$ ) that would disfavour binding of a larger negatively charged pTyr. Thus Y717 seems highly unlikely to be phosphorylated after Y715, since if pY715 cannot enter this pocket then the correct positioning of $\mathrm{Y} 717$ at the active site would also be disrupted (Fig. 2f). A similar logic applies when considering Y713 and Y715, phosphorylation of Y713 follows phosphorylation of Y715. The most abundant $5 \mathrm{P}$ and $4 \mathrm{P}$ peptides possess pY708, pY713, pY715, pY717, in a pattern predicted by structural analysis to arise from sequential C-terminal phosphorylation. This is consistent with mass spectrometry (Supplementary Fig. 2a). As the peptide passes through the active site, the surface area buried by the interaction of the tyrosine-rich peptide and the kinase domain progressively decreases, destabilising the

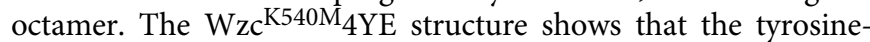
rich tail would be accessible to the cognate phosphatase Wzb (Fig. 4d) $)^{9,29,30}$. Wzc would be expected to constantly cycle between phosphorylated monomers and dephosphorylated octamers in the cell, consistent with the requirement for both kinase and phosphatase activity ${ }^{13-15}$.

Several proteins in the synthetic machinery of Gram-positive bacteria have been reported to be activated by PCP-2b-mediated phosphorylation (reviewed in ${ }^{1,31}$ ). Tyrosine phosphopeptides have been identified in enzymes involved in the Gram-negative Klebsiella capsule biosynthesi ${ }^{32}$, and there are differing reports on Wzc-mediated phosphorylation of the biosynthetic uridine diphosphoglucose dehydrogenase in $E$. coli ${ }^{33,34}$. This suggests that monomeric Wzc, with its accessible active site, may exert additional regulatory effects upon bacterial metabolism.

Available data indicates that Wzc engages with Wza during export and synthesis. This evidence can be summarised as (i) genetic experiments support cognate recognition of Wza-Wzc partners ${ }^{21}$; (ii) Wza-Wzc heterocomplexes have been observed by microscopy ${ }^{22}$; (iii) deletion of $w z a$ prevents polymer production in a phenotype identical to $w z c$-deletions, suggesting an indirect (feedback) on the Wzy polymerase ${ }^{35}$. In purified Wza octamers, the OM channel is closed to the periplasm by a periplasmic tyrosine ring ${ }^{19,36}$ and this must be opened for translocation as the polysaccharide passes through its central cavity ${ }^{20}$. Structural analysis of Wzc identifies the large periplasmic helical bundle (motif 3) (Fig. 2a) as the candidate for interaction with the periplasmic portion of octameric translocase Wza. Motif 3 is absent in Gram-positive bacteria (Supplementary Fig. 5b) which, of course, lack an OM. In addition, Wzz (which lacks an OM protein partner) has an entirely different helical structure from 


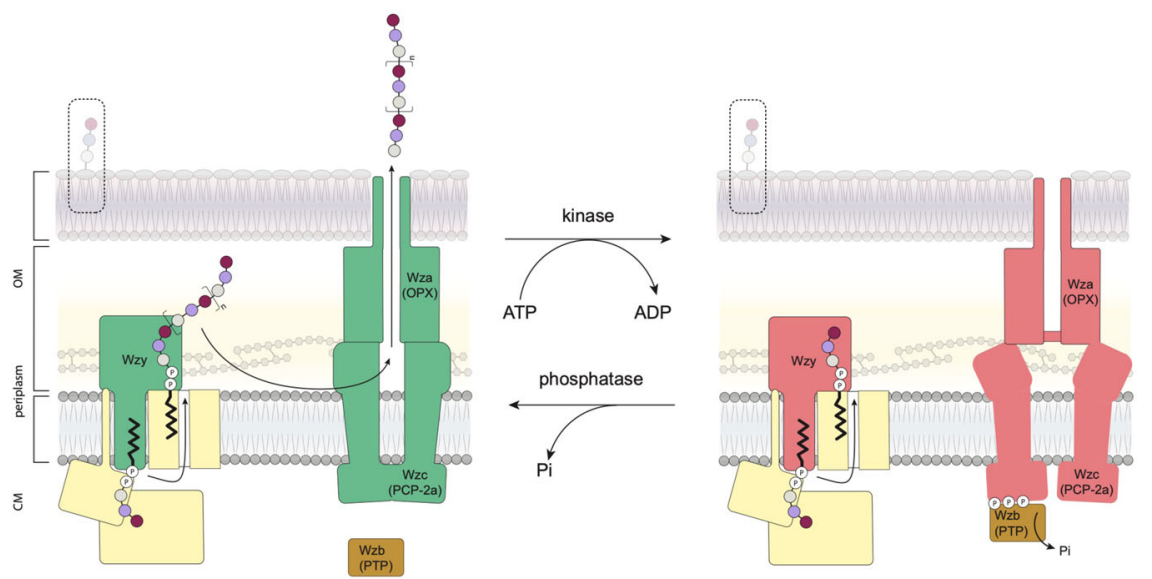

Fig. 6 Model for Wzc function. Wzc, the master regulator of capsule assembly and export regulates the activity of Wzy (polymerase) and opens Wza (translocon). Multi phosphorylated Wzc is found as a monomer whilst dephosphorylated Wzc forms an octamer. Capsule product and export requires continuous Wzc cycling between phosphorylation and dephosphorylation states. Polymer chain completion may be accomplished in a single or stepwise by multiple cycles.

Wzc motif 3 (Fig. 3f). Deletion of the periplasmic helices in motif 3 resulted in a Wzc variant protein that retained the ability to form octamers and possessed normal autokinase activity (Fig. 5a, b). However, the mutant abolished the production of polysaccharides uncoupling these activities from phosphorylation (Fig. 5b). This phenotypic outcome resembles deletion of Wza in vivo ${ }^{35}$. The data is consistent with these helices being key to the control of Wza and the interaction of $\mathrm{Wzc}$ with $\mathrm{Wza}$ being required for activation of Wzy.

We propose that the machine that drives synthesis and export of EPS is a ternary complex minimally comprising octameric Wzc, octameric open-form Wza and Wzy. Autophosphorylation of Wzc results in octamer dissociation, leading to the dissolution of the machine which halts polymerisation and closes Wza (Fig. 6). The capacity to reassemble the machine for renewed synthesis and export, therefore, depends on the dephosphorylation of $\mathrm{Wzc}$ and rationalises the essential requirement for Wzb.

The molecular insights into this system provide the essential foundation to investigate these predictions and offer opportunities for efforts to target polysaccharide biosynthesis for both therapeutic and industrial benefit.

\section{Methods}

Cloning, expression and purification of Wzc. The $w z c$ gene from E. coli E69 (O9a:K30) gene was cloned into vector pBAD24 ${ }^{37}$ to generate pBRC901, in order to express Wzc with a C-terminal hexa-histidine tag. E.coli TOP 10 cells transformed with pBRC901 were grown at $37^{\circ} \mathrm{C}$ until $\mathrm{OD}_{600}$ reached approximately 0.8 , then Wzc expression was induced with $0.002 \%$ arabinose at $20^{\circ} \mathrm{C}$ overnight. Cells were harvested by centrifugation and stored at $-80^{\circ} \mathrm{C}$. Cell pellets were resuspended with lysis buffer $(20 \mathrm{mM} \mathrm{Na}$ phosphate, pH7.0, $500 \mathrm{mM} \mathrm{NaCl})$ and lysed by passage through a Constant Systems cell disruptor. Unbroken cells were removed by centrifugation at $20,000 \times g$ for $1 \mathrm{~h}$ at $4{ }^{\circ} \mathrm{C}$. Membranes were collected by ultracentrifugation at $186,000 \times g$ for $1 \mathrm{~h}$ at $4^{\circ} \mathrm{C}$ and solubilized with $20 \mathrm{mM} \mathrm{Na}$ phosphate, pH7.0, $500 \mathrm{mM} \mathrm{NaCl}, 1 \% \mathrm{DDM}$ at $4{ }^{\circ} \mathrm{C}$. Residual membrane debris was removed by ultracentrifugation and the supernatant containing Wzc was purified under gravity using ABT nickel resin (Cat. No. 6BCL-NTANi-100). The protein was eluted with $20 \mathrm{mM}$ Na phosphate, pH 7.0, $500 \mathrm{mM} \mathrm{NaCl}, 0.003 \%$ Lauryl Maltose Neopentyl Glycol (LMNG) containing $300 \mathrm{mM}$ imidazole after successive column washes with $20 \mathrm{mM}$ imidazole and $50 \mathrm{mM}$ imidazole. Purified protein was buffer exchanged to buffer ( $20 \mathrm{mM}$ Na phosphate, $\mathrm{pH} 7.0,500 \mathrm{mM} \mathrm{NaCl}, 0.003 \%$ Lauryl Maltose Neopentyl Glycol (LMNG)) using a CentriPure P100 column and concentrated with a $100 \mathrm{kDa}$ concentrator. Concentrated protein was applied to a Superose $610 / 300$ increase column equilibrated with $20 \mathrm{mM}$ HEPES, $150 \mathrm{mM}$ $\mathrm{NaCl}, 0.001$ \% LMNG, 2 mM tris(2-carboxyethyl)phosphine (TECP), pH 7.3. Wzc mutant derivatives were made through site-directed mutagenesis or by Gibson assembly following the manufacture's protocols (New England Biolabs). Expression and purification of Wzc mutants were performed as described for the native protein.
For the dephosphorylation, Wzc was incubated with $\mathrm{Wzb}^{9}$ at room temperature in a molar ratio of 1:5. Samples were taken after $0,1 \mathrm{~h}, 2 \mathrm{~h}$ and $3 \mathrm{~h}$ intervals and the presence of phosphorylation on tyrosine were monitored as described below.

Western immunoblot detection of phosphorylated Wzc proteins. In total, $8 \mu \mathrm{l}$ of $0.2 \mathrm{mg} / \mathrm{ml}$ purified proteins were analyzed by western immunoblotting using mouse monoclonal anti-pTyr antibody (Sigma, Cat. No. P4110, dilution 1:5000) as primary and an HRP conjugated anti-mouse IgG antibody (Promega, Cat. No. W402B, dilution 1:5000) as secondary antibody. An anti-polyHistidine-peroxidase antibody (Sigma, Cat. No. A7058, dilution 1:5000) was used to detect hexahistidine tagged Wzc protein, for showing the loading amount.

Wzc variants were also examined in whole-cell lysates prepared from $E$. coli CWG285 transformants grown in LB medium (containing $100 \mu \mathrm{g} / \mathrm{ml}$ ampicillin where required) at $37^{\circ} \mathrm{C}$. CWG285 is derived from E. coli E69 and contains one insertion in $w z c$, and another insertion that exerts a polar inactivating effect on the $w z c$ homolog, etk $k^{13}$. Lysates were prepared by harvesting $1 \mathrm{OD}_{600 \mathrm{~nm}}$ equivalent of bacteria by centrifugation at $12,000 \times g$ for $2 \mathrm{~min}$. The pellets were lysed in $100 \mu \mathrm{L}$ of Laemmli buffer (125 mM Tris-Cl, pH 6.8, $20 \%$ (w/v) glycerol, 4\% (w/v) SDS, $0.004 \%(\mathrm{w} / \mathrm{v})$ bromophenol blue $)^{38}$ and heated at $100{ }^{\circ} \mathrm{C}$ for $10 \mathrm{~min}$. The wholecell lysates were analysed by SDS-PAGE and transferred to Protran $0.45 \mathrm{im}$ nitrocellulose membrane (Amersham). Hexa-histidine-tagged Wzc was identified using mouse anti-pentaHis antibody (Qiagen, 1:2000) and phosphorylated Wzc was detected with mouse PY20 antibody (Sigma, Cat. No. P4110, 1:2000). Goat anti-mouse horseradish peroxidase (HRP) conjugated antibody (Cedar Lane, 1:3000) was used as the secondary antibody. HRP was detected by chemiluminescence using the Crescendo Western HRP substrate (Millipore). The uncropped images of gels are available in Supplementary Fig. 10.

Western immunoblot analysis of cell surface polysaccharides. Wzc variants were tested for function by transforming the corresponding plasmids into $E$. coli CWG285. Bacteria were grown and whole-cell lysates prepared as described above, with the exception that samples of CPS analysis were treated with proteinase $\mathrm{K}(0.5$ $\mathrm{mg} / \mathrm{mL}$ ) at $55^{\circ} \mathrm{C}$ for $1 \mathrm{~h}^{39}$. The whole-cell lysates were separated and transferred to nitrocellulose as described above. K30 CPS was detected using rabbit anti-K30 antiserum ${ }^{40}$ as the primary antibody at a 1:3000 dilution and goat anti-rabbit alkaline phosphatase (AP) conjugated antibody (Cedar Lane, 1:3000) as the secondary antibody. AP was detected with nitro blue tetrazolium and 5-bromo-4chloro-3-indolyl phosphate (Roche).

Native mass spectrometry. Prior to MS analysis, the samples were buffer exchanged into $200 \mathrm{mM}$ ammonium acetate $\mathrm{pH} 8.0$ and $2 \times$ critical micelle concentration of detergent (C8E4 and LDAO) using Biospin-6 (BioRad) column and introduced directly into the mass spectrometer using gold-coated capillary needles (prepared in-house). Data were collected on a Q-Exactive UHMR mass spectrometer (ThermoFisher). The instrument parameters were as follows: capillary voltage $1.2 \mathrm{kV}$, quadrupole selection from 1000 to $20,000 \mathrm{~m} / \mathrm{z}$ range, $\mathrm{S}$-lens $\mathrm{RF} 100 \%$, collisional activation in the HCD cell $200-300 \mathrm{~V}$, trapping gas pressure setting 7.5, temperature $250^{\circ} \mathrm{C}$, resolution of the instrument 12,500 . The noise level was set at 3 rather than the default value of 4.64 . No in-source dissociation was applied. Data were analyzed using Xcalibur 4.2 (Thermo Scientific) software package. All experiments were repeated three times with similar outcomes. 
Phosphoproteomics and phosphopeptide analysis. For phosphorylation identification, the tryptic peptides were loaded onto a reverse phase C18 trap column (Acclaim PepMap 100, $75 \mu \mathrm{m} \times 2 \mathrm{~cm}$, nano viper, C18, $3 \mu \mathrm{m}, 100 \AA$, ThermoFisher, Waltham, MA, U.S.A) using an Ultimate 3000 and washed with $50 \mu \mathrm{L}$ of $0.1 \%$ formic acid at $10 \mu \mathrm{l} / \mathrm{min}$. The desalted peptides were then separated using a $15 \mathrm{~cm}$ pre-packed reverse-phase analytical column (Acclaim PepMap 100, $75 \mu \mathrm{m} \times 15 \mathrm{~cm}$, C18, $3 \mu \mathrm{m}, 100 \AA$, ThermoFisher, Waltham, MA, U.S.A) using a 45 min linear gradient from 5 to $40 \%$ eluant ( $80 \%$ acetonitrile, $20 \%$ water, $0.1 \%$ formic acid) at a flow rate of $300 \mathrm{~nL} / \mathrm{min}$. The separated peptides were electrosprayed into an Orbitrap Eclipse Tribrid mass spectrometry system in the positive ion mode using data-dependent acquisition with a $3 \mathrm{~s}$ cycle time. Precursors and products were detected in the Orbitrap analyzer at a resolving power of 60,000 and 30,000 (@ m/z 200 ), respectively. Precursor signals with an intensity $>1.0 \times 10^{-4}$ and charge state between 2 and 7 were isolated with the quadrupole using a $0.7 \mathrm{~m} / \mathrm{z}$ isolation window $(0.5 \mathrm{~m} / \mathrm{z}$ offset) and subjected to MS/MS fragmentation using higher energy collision-induced dissociation (30\% relative fragmentation energy). MS/MS scans were collected at an AGC setting of $1.0 \times 10^{4}$ or a maximum fill time of 100 $\mathrm{ms}$ and precursors within $10 \mathrm{ppm}$ were dynamically excluded for $30 \mathrm{~s}$. Raw data files were processed using MaxQuant (version 1.6.3.4) and searched against the Escherichia coli (O9a:K30) biosynthesis gene cluster ${ }^{30}$ as well as a list of common contaminants using the Andromeda search engine ${ }^{41,42}$ with the following search parameters: trypsin digestion; fixed modification was set to carbamidomethyl (C); variable modifications set as oxidation $(\mathrm{M})$, acetylated protein $\mathrm{N}$ terminus, and phosphorylation $(\mathrm{Y})$; up to two missed cleavages allowed. Mass spectra were recalibrated within MaxQuant with a precursor error tolerance of 20 parts per million (ppm) and then re-searched with a mass tolerance of $5 \mathrm{ppm}$. Note that our initial analyses with pSYTH search yielded the assignment of phosphosites on His and Ser along with the conserved Tyr residues within the peptide 708-718 given their close proximity, however, based on manual inspection and phosphoproteomics analyses on several mutants (data is on PRDIE with accession number PXD025820) suggested that the assignment on His and Ser was not correct. Thus, we used pY only for searching phosphosites in our subsequent analyses.

EM analysis. For negative stain, $3.5 \mu \mathrm{l}$ of purified protein (around $0.05 \mathrm{mg} / \mathrm{ml}$ ) was applied to glow-discharged 400-mesh carbon-coated copper grids, which were then washed three times with $\mathrm{ddH}_{2} \mathrm{O}$ and stained with $0.75 \%$ uranyl formate. Images were acquired using an FEI Tecnai T12 microscope at a magnification of $\times 42,000$, with a calibrated pixel size of $2.63 \AA$.

For cryo-EM of Wzc ${ }^{\mathrm{K} 540 \mathrm{M}}$ and Wzc ${ }^{\mathrm{K} 540 \mathrm{M}} 4 \mathrm{YE}, 3.5 \mu \mathrm{l}$ purified sample at $\sim 2 \mathrm{mg} /$ $\mathrm{ml}$ was applied to glow-discharged Quantifoil gold R1.2/1.3 300-mesh grids and the grids were blotted for about $3 \mathrm{~s}$ in conditions of $100 \%$ humidity and $4{ }^{\circ} \mathrm{C}$ before vitrification in liquid ethane using Vitrobot (FEI). All datasets were collected on a Titan Krios equipped with a K3 direct electron detector at eBIC, Diamond Light Source, UK. The apo- and ADP-complex datasets were collected by SerialEM at a magnification of $\times 105,000$ with the physical pixel size of $0.829 \AA /$ pixel. The total dose was $55 \mathrm{e} / \AA^{2}$ and $53.6 \mathrm{e} / \AA^{2}$ for apo- and ADP-complex datasets, respectively, and the defocus range was $-0.5 \mu \mathrm{m}$ to $-2.5 \mu \mathrm{m}$. The $4 \mathrm{YE}$ dataset was collected by EPU software at a magnification of $\times 105,000$, with the physical pixel size $0.831 \AA /$ pixel, using a defocus range of $-0.5 \mu \mathrm{m}$ to $-3.0 \mu \mathrm{m}$. The total dose was $57.5 \mathrm{e} / \AA^{2}$.

All EM movies were motion-corrected by MotionCor $2^{43}$ through Relion3.0. Contrast transfer function values were estimated by Gctf ${ }^{44}$ or CTFFIND ${ }^{45}$. Particles were picked using Laplacian-of-Gaussian (LoG), based auto-picking in Relion3. $0^{46}$. After extraction and normalization in Relion3.0, particles were imported into cryoSPARC ${ }^{47}$ for further processing. Multiple rounds of $2 \mathrm{D}$ classification were carried out. The initial model was generated from a selection of $2 \mathrm{D}$ classes in cryoSPARC ${ }^{47}$. Other rounds of $3 \mathrm{D}$ classifications were carried out without applying symmetry. C8 symmetry was observed for the resulting maps, except motif 3 . For the ADP-complex and $4 \mathrm{YE}$ mutant maps, the best $3 \mathrm{D}$ classes were reconstructed using non-uniform refinement ${ }^{48}$ in cryoSPARC applying $\mathrm{C} 1$ or $\mathrm{C} 8$ symmetries. For the apo-structure, after cleaning through multiple rounds of 2D and 3D classification, 673,112 particles were used for further 3D classification with $\mathrm{C} 1$ symmetry. One representative class with the most complete motif 3 was chosen for non-uniform refinement to generate a $2.85 \AA$ map at C1 symmetry. A mask covering only the periplasmic region was generated and local refinement was carried out in cryoSPARC ${ }^{47}$. The resulting $2.77 \AA$ map showed clear density for the side chains of the periplasmic motif 3 . For the $\mathrm{C} 8$ reconstruction, further $3 \mathrm{D}$ classifications were carried out and 546,088 particles were subjected to one final round of non-uniform refinement with C8 symmetry to yield a $2.3 \AA$ map (Supplementary Fig. 3a)

For Wzb treated Wzc, $3.5 \mu \mathrm{l}$ of purified sample at $\sim 1.8 \mathrm{mg} / \mathrm{ml}$ was used to make the grids in the same way described above. In total, 1787 micrographs were collected on Glacios (Thermofisher) at a magnification of $\times 92,000$ with the physical pixel size $1.55 \AA$ \&ixel. Defocus range was $-1.0 \mu \mathrm{m}$ to $-2.5 \mu \mathrm{m}$. Total dose was 60 $\mathrm{e} / \AA^{2}$. Using convolutional neural networks based algorithm Topaz ${ }^{23}$ we were able to pick 356,511 particles. Multiple rounds of 2D classifications were carried out, and the class averages showing clear Wzc octameric end views were selected and analysed.

Grids of $\mathrm{Wzc}^{\mathrm{K} 540 \mathrm{M}} \Delta \mathrm{Motif} 3$ at $\sim 1.9 \mathrm{mg} / \mathrm{ml}$ were made in the same way described above. Dataset was collected on Glacios at a magnification of $\times 92,000$ with the physical pixel size $1.55 \AA /$ pixel. The total dose was $60.38 \mathrm{e} / \AA^{2}$. Data was processed similar to $\mathrm{Wzc}^{\mathrm{K} 540 \mathrm{M}}$ to get $2 \mathrm{D}$ class averages.

Model building and refinement. For the apo-structure, the cytoplasmic kinase domain was refined from a homology model (PDB code 3LA6). The other regions were built manually in $\operatorname{Coot}^{49}$. The $2.3 \AA \mathrm{C} 8$-symmetry map was used to build the structure, with the exception of motif 3 . The locally refined $2.77 \AA \mathrm{C} 1$ symmetry map was used to build the three classes of motif 3 . The entire structure was assembled in Coot and refined against the $2.85 \AA \mathrm{C} 1$ map. The structure without motif 3 was refined against the C8 map. For the ADP-bound complex structure, ligands were manually added in Coot and the structure was refined in PHENIX ${ }^{50}$ For the 4YE mutant structure, the mutation points were manually built in Coot, based on the apo-structure, and refinement was carried out in PHENIX. Figures of cryo-EM maps and structures were generated with PyMOL (The PyMOL Molecular Graphics System, Version 2.1 Schrödinger, LLC) and UCSF Chimera ${ }^{51}$.

Antibodies. Monoclonal anti-polyHistidine-peroxidase antibody produced in mouse was purchased from Sigma (Cat. No. A7058). Monoclonal antiphosphotyrosine antibody produced in mouse was purchased from Sigma (Cat. No P4110). HRP conjugated anti-mouse IgG $(\mathrm{H}+\mathrm{L})$ antibody was purchased from Promega and used as the secondary antibody for detecting phosphotyrosine (Cat. No.W402B). AP-conjugated goat anti-rabbit IgG was purchased from Cedarlane (Cat. No. CLCC43008). Peroxidase-conjugated goat anti-mouse IgG was purchased from Cedarlane (Cat. No. 115-036-003). Penta-his antibody was purchased from Qiagen (Cat. No. 34660)

Reporting summary. Further information on research design is available in the Nature Research Reporting Summary linked to this article.

\section{Data availability}

The data that supports this study are available from the corresponding authors upon reasonable request. EM maps and models are deposited in the EMDB and wwPDB under accession codes EMD-12338 and PDB 7NHR (C1 WzcK540M); EMD-12339 and PDB 7NHS (C8 WzcK540M); EMD-12340 (WzcK540M periplasmic localized map); EMD12360 and PDB 7NII (C1 WzcK540M ADP complex); EMD-12359 and PDB 7NIH (C8 WzcK540M ADP complex); EMD-12353 and PDB 7NIB (C1 WzcK540M4YE); EMD12349 and PDB 7NI2 (C8 WzcK540M4YE). The mass spectrometry proteomics data have been deposited to the ProteomeXchange Consortium via the PRIDE partner repository with the dataset identifier PXD025820. All constructs are available from the authors upon request until their deposition and release by ADDGENE. Source data are provided with this paper.

Received: 12 March 2021; Accepted: 29 June 2021; Published online: 16 July 2021

\section{References}

1. Whitfield, C., Wear, S. S. \& Sande, C. Assembly of bacterial capsular polysaccharides and exopolysaccharides. Annu. Rev. Microbiol. 74, 521-543 (2020).

2. Morona, R., Purins, L., Tocilj, A., Matte, A. \& Cygler, M. Sequence-structure relationships in polysaccharide co-polymerase (PCP) proteins. Trends Biochem. Sci. 34, 78-84 (2009).

3. Jadeau, F. et al. BYKdb: the bacterial protein tYrosine kinase database. Nucleic Acids Res. 40, D321-D324 (2012).

4. Whitfield, C., Williams, D. M. \& Kelly, S. D. Lipopolysaccharide O-antigensbacterial glycans made to measure. J. Biol. Chem. 295, 10593-10609 (2020).

5. Wiseman, B., Nitharwal, R. G., Widmalm, G. \& Högbom, M. Structure of a full-length bacterial polysaccharide co-polymerase. Nat. Commun. 12, 369 (2021)

6. Collins, R. F. et al. Full-length, oligomeric structure of Wzz determined by cryoelectron microscopy reveals insights into membrane-bound states. Structure 25, 806-815.e3 (2017).

7. Kalynych, S., Cherney, M., Bostina, M., Rouiller, I. \& Cygler, M. Quaternary structure of WzzB and WzzE polysaccharide copolymerases. Protein Sci. 24, 58-69 (2015)

8. Lescop, E. et al. The solution structure of Escherichia coli Wzb reveals a novel substrate recognition mechanism of prokaryotic low molecular weight protein-tyrosine phosphatases. J. Biol. Chem. 281, 19570-19577 (2006).

9. Hagelueken, G., Huang, H., Mainprize, I. L., Whitfield, C. \& Naismith, J. H. Crystal structures of Wzb of Escherichia coli and CpsB of Streptococcus pneumoniae, representatives of two families of tyrosine phosphatases that regulate capsule assembly. J. Mol. Biol. 392, 678-688 (2009). 
10. Ilan, O. et al. Protein tyrosine kinases in bacterial pathogens are associated with virulence and production of exopolysaccharide. EMBO J. 18, 3241-3248 (1999).

11. Vincent, C. et al. Relationship between exopolysaccharide production and protein-tyrosine phosphorylation in gram-negative bacteria. J. Mol. Biol. 304, 311-321 (2000).

12. Doublet, P., Grangeasse, C., Obadia, B., Vaganay, E. \& Cozzone, A. J. Structural organization of the protein-tyrosine autokinase Wzc within Escherichia coli cells. J. Biol. Chem. 277, 37339-37348 (2002).

13. Wugeditsch, T. et al. Phosphorylation of Wzc, a tyrosine autokinase, is essential for assembly of group 1 capsular polysaccharides in Escherichia coli. J. Biol. Chem. 276, 2361-2371 (2001).

14. Obadia, B. et al. Influence of tyrosine-kinase Wzc activity on colanic acid production in Escherichia coli K12 cells. J. Mol. Biol. 367, 42-53 (2007).

15. Nadler, C. et al. Cycling of Etk and Etp phosphorylation states is involved in formation of group 4 capsule by Escherichia coli. PLoS ONE 7, e37984 (2012).

16. Bechet, E. et al. Identification of structural and molecular determinants of the tyrosine-kinase Wzc and implications in capsular polysaccharide export. Mol. Microbiol. 77, 1315-1325 (2010).

17. Olivares-Illana, V. et al. Structural basis for the regulation mechanism of the tyrosine kinase CapB from Staphylococcus aureus. PLoS Biol. 6, e143 (2008).

18. Lee, D. C., Zheng, J., She, Y. M. \& Jia, Z. Structure of Escherichia coli tyrosine kinase Etk reveals a novel activation mechanism. EMBO J. 27, 1758-1766 (2008).

19. Dong, C. J. et al. Wza the translocon for E. coli capsular polysaccharides defines a new class of membrane protein. Nature 444, 226-229 (2006).

20. Nickerson, N. N. et al. Trapped translocation intermediates establish the route for export of capsular polysaccharides across Escherichia coli outer membranes. Proc. Natl Acad. Sci. USA 111, 8203-8208 (2014).

21. Reid, A. N. \& Whitfield, C. functional analysis of conserved gene products involved in assembly of Escherichia coli capsules and exopolysaccharides: evidence for molecular recognition between Wza and Wzc for colanic acid biosynthesis. J. Bacteriol. 187, 5470-5481 (2005)

22. Collins, R. F. et al. The $3 \mathrm{D}$ structure of a periplasm-spanning platform required for assembly of group 1 capsular polysaccharides in Escherichia coli. Proc. Natl Acad. Sci. USA 104, 2390-2395 (2007).

23. Bepler, T. et al. Positive-unlabeled convolutional neural networks for particle picking in cryo-electron micrographs. Nat. Methods 16, 1153-1160 (2019).

24. Paiment, A., Hocking, J. \& Whitfield, C. Impact of phosphorylation of specific residues in the tyrosine autokinase, Wzc, on its activity in assembly of group 1 capsules in Escherichia coli. J. Bacteriol. 184, 6437-6447 (2002).

25. Ernst, C. M. et al. Adaptive evolution of virulence and persistence in carbapenem-resistant Klebsiella pneumoniae. Nat. Med. 26, 705-711 (2020).

26. Talyansky, Y. et al. Capsule carbohydrate structure determines virulence in Acinetobacter baumannii. PLoS Pathog. 17, e1009291 (2021).

27. Dams-Kozlowska, H. \& Kaplan, D. L. Protein engineering of wzc to generate new emulsan analogs. Appl. Environ. Microbiol. 73, 4020-4028 (2007).

28. Leo, V., Tran, E. \& Morona, R. Polysaccharide co-polymerase WzzB/WzzE chimeras reveal transmembrane 2 region of WzzB is important for interaction with WzyB. J. Bacteriol. (2020).

29. Temel, D. B., Dutta, K. \& Ghose, R. Sequence-specific backbone ${ }^{1} \mathrm{H},{ }^{13} \mathrm{C}$ and ${ }^{15} \mathrm{~N}$ assignments of the catalytic domain of the Escherichia coli protein tyrosine kinase, Wzc. Biomol. NMR Assign. 8, 37-41 (2014).

30. Drummelsmith, J. \& Whitfield, C. Gene products required for surface expression of the capsular form of the group $1 \mathrm{~K}$ antigen in Escherichia coli (O9a:K30). Mol. Microbiol. 31, 1321-1332 (1999).

31. Mijakovic, I. \& Macek, B. Impact of phosphoproteomics on studies of bacterial physiology. FEMS Microbiol. Rev. 36, 877-892 (2012).

32. Lin, M. H. et al. Phosphoproteomics of Klebsiella pneumoniae NTUH-K2044 reveals a tight link between tyrosine phosphorylation and virulence. Mol. Cell Proteom. 8, 2613-2623 (2009).

33. Lacour, S., Bechet, E., Cozzone, A. J., Mijakovic, I. \& Grangeasse, C. Tyrosine phosphorylation of the UDP-glucose dehydrogenase of Escherichia coli is at the crossroads of colanic acid synthesis and polymyxin resistance. PLoS ONE 3, e3053 (2008).

34. Mainprize, I. L., Bean, J. D., Bouwman, C., Kimber, M. S. \& Whitfield, C. The UDP-glucose dehydrogenase of Escherichia coli K-12 displays substrate inhibition by NAD that is relieved by nucleotide triphosphates. J. Biol. Chem. 288, 23064-23074 (2013).

35. Drummelsmith, J. \& Whitfield, C. Translocation of group 1 capsular polysaccharide to the surface of Escherichia coli requires a multimeric complex in the outer membrane. EMBO J. 19, 57-66 (2000).

36. Kong, L., Almond, A., Bayley, H. \& Davis, B. G. Chemical polyglycosylation and nanolitre detection enables single-molecule recapitulation of bacterial sugar export. Nat. Chem. 8, 461-469 (2016).

37. Guzman, L. M., Belin, D., Carson, M. J. \& Beckwith, J. Tight regulation, modulation, and high-level expression by vectors containing the arabinose PBAD promoter. J. Bacteriol. 177, 4121-4130 (1995).
38. Laemmli, U. K. Cleavage of structural proteins during the assembly of the head of bacteriophage T4. Nature 227, 680-685 (1970).

39. Hitchcock, P. J. \& Brown, T. M. Morphological heterogeneity among Salmonella lipopolysaccharide chemotypes in silver-stained polyacrylamide gels. J. Bacteriol. 154, 269-277 (1983).

40. Dodgson, C., Amor, P. \& Whitfield, C. Distribution of the rol gene encoding the regulator of lipopolysaccharide O-chain length in Escherichia coli and its influence on the expression of group I capsular K antigens. J. Bacteriol. 178, 1895-1902 (1996).

41. Cox, J. et al. Andromeda: a peptide search engine integrated into the MaxQuant environment. J. Proteome Res. 10, 1794-1805 (2011).

42. Cox, J. \& Mann, M. MaxQuant enables high peptide identification rates, individualized p.p.b.-range mass accuracies and proteome-wide protein quantification. Nat. Biotechnol. 26, 1367-1372 (2008).

43. Zheng, S. Q. et al. MotionCor2: anisotropic correction of beam-induced motion for improved cryo-electron microscopy. Nat. Methods 14, 331-332 (2017).

44. Zhang, K., Gctf \& Real-time, C. T. F. Determination and correction. J. Struct. Biol. 193, 1-12 (2016).

45. Rohou, A. \& Grigorieff, N. CTFFIND4: fast and accurate defocus estimation from electron micrographs. J. Struct. Biol. 192, 216-221 (2015).

46. Scheres, S. H. RELION: implementation of a Bayesian approach to cryo-EM structure determination. J. Struct. Biol. 180, 519-530 (2012).

47. Punjani, A., Rubinstein, J. L., Fleet, D. J. \& Brubaker, M. A. cryoSPARC: algorithms for rapid unsupervised cryo-EM structure determination. Nat. Methods 14, 290-296 (2017).

48. Punjani, A., Zhang, H. \& Fleet, D. J. Non-uniform refinement: adaptive regularization improves single-particle cryo-EM reconstruction. Nat. Methods 17, 1214-1221 (2020).

49. Emsley, P. \& Cowtan, K. Coot: model-building tools for molecular graphics. Acta Crystallogr. Sect. D. Biol. Crystallogr. 60, 2126-2132 (2004).

50. Adams, P. D. et al. PHENIX: a comprehensive python-based system for macromolecular structure solution. Acta Crystallogr D. Biol. Crystallogr 66, 213-221 (2010).

51. Pettersen, E. F. et al. UCSF chimera-a visualization system for exploratory research and analysis. J. Comput. Chem. 25, 1605-1612 (2004).

\section{Acknowledgements}

J.H.N., Y.Y., and P.W. are supported by Wellcome Trust (100209/Z/12/Z). J.R.B. and C. V.R. are supported by MRC Programme grants MR/N020413/1 and MR/V028839/1 where J.R.B. is a Researcher Co-Investigator on the latter. We acknowledge the Wellcome Trust Membrane Protein Laboratory (20289/Z16/Z) for support. C.W. is the recipient of a Canada Research Chair and his research (and that of L.S. and B.R.C) is supported by a Canadian Institutes of Health Research Foundation grant (FDN-2016-148364 to CW). J. L. and P.Z. are supported by Wellcome Trust Investigator Award (206422/Z/17/Z). We acknowledge Diamond for access and support of the CryoEM facilities at the UK national electron bio-imaging centre (eBIC, proposal EM20223), funded by the Wellcome Trust, MRC and BBSRC. All grids pre-screened and selected using the cryo-EM facility (OPIC) in the Division of Structural Biology, University of Oxford, part of the UK Centre of Instruct-ERIC. The Franklin is a core funded research Institute of the EPSRC.

\section{Author contributions}

Y.Y. purified proteins with contributions from P.N.W., Y.Y., J.L., P.Z., and J.H.N. carried out the E.M. structural studies and analysis. B.C., L.S., and C.W. carried out the in vivo assay and analysis of Wzc mutant function. J.B. and C.V.R. carried out the mass spectrometry. J.H.N. and C.W. led the study. All authors contributed to the analysis of data and writing of the manuscript.

\section{Competing interests}

The authors declare no competing interests.

\section{Additional information}

Supplementary information The online version contains supplementary material available at https://doi.org/10.1038/s41467-021-24652-1.

Correspondence and requests for materials should be addressed to C.W. or J.H.N.

Peer review information Nature Communications thanks Albert Heck, Benjamin Wiseman and the other, anonymous, reviewer(s) for their contribution to the peer review of this work. Peer reviewer reports are available.

Reprints and permission information is available at http://www.nature.com/reprints

Publisher's note Springer Nature remains neutral with regard to jurisdictional claims in published maps and institutional affiliations. 
(c) (i) Open Access This article is licensed under a Creative Commons Attribution 4.0 International License, which permits use, sharing, adaptation, distribution and reproduction in any medium or format, as long as you give appropriate credit to the original author(s) and the source, provide a link to the Creative Commons license, and indicate if changes were made. The images or other third party material in this article are included in the article's Creative Commons license, unless indicated otherwise in a credit line to the material. If material is not included in the article's Creative Commons license and your intended use is not permitted by statutory regulation or exceeds the permitted use, you will need to obtain permission directly from the copyright holder. To view a copy of this license, visit http://creativecommons.org/ licenses/by/4.0/.

(C) The Author(s) 2021 